\title{
Local Reinforcement in Notched Fiber Metal Laminates by Bonding Titanium Patch
}

\author{
Jipeng Zhang, Yue Wang, Jiazhen Zhang and Zhengong Zhou \\ Center for Composite Materials and Structures, Harbin Institute of Technology, Harbin 150001, China
}

\begin{abstract}
In order to reduce the stress concentration in notched fiber metal laminate (FML) and improve its damage tolerance, the local reinforcement concept was employed in present paper. Two different reinforced structures were proposed by bonding titanium patch on single side or double sides of the standard glass fiber reinforced aluminum laminate (GLARE). Tensile tests were conducted on these reinforced GLARE, and their mechanical properties were compared for evaluating the reinforced effect of titanium patch, as well as the damage behavior which dominated the mechanical response was analysed later to capture the failure mechanism of the reinforced GLARE. The reinforced effect of titanium patch on mechanical response of GLARE laminate was usually characterized by the improvements of modulus and yield strength, as well as the significant increase of ultimate strain and strength. Cohesive failure and adhesive failure were detected in the adhesive between titanium patch and GLARE laminate, where the adhesive failure corresponded to the weak reinforced case. In addition, the variation of delamination in GLARE laminate was also closely related to the reinforced effect on mechanical response. It is expected that the present investigation can provide technical support for damage tolerance design of fiber metal laminates.
\end{abstract}

\section{Introduction}

With the increasing requirements of damage tolerance in aeronautics, FMLs which consist of thin metal sheets and fiber reinforced polymer composites have gained more attention, especially after the successful application of GLARE in the A380 aircraft [1]. The FML concept was originally proposed for fatigue critical applications. While with its development, some potential advantages over the conventional metal alloys and fiber reinforced composites in structural components applications have also been recognized, which usually in terms of the high strength and excellent resistance to impact, corrosion and moisture [2-5].

In structural design, the blunt notches are usually inevitable, which usually come in the form of rivet holes or bolt holes. The mechanical behavior of structure components with holes should be paid particular attentions, since the undesired stress concentration and its accompanying properties degradation or even premature failure possibilities will constitute a challenge to the service life. A few investigations have been carried out to evaluate the notched behavior of FMLs. The effects of notch geometry and size on notched strength and notch sensitivity were investigated experimentally, from which it was concluded that the sizing effect was more significant [6, 7]. Moreover some notched FMLs have also been tested under off-axis loading, in which the offaxis dependence of notched behavior was evaluated $[8,9]$. Except the experimental investigations, numerical simulations were also performed on the notched FMLs, where the damage patterns of the constituents in FMLs and their corresponding damage evolution processes were analysed [10-12].

The damage tolerance design requires the damaged structural components to sustain the limit load in service life, and then the reinforcement concept should be paid more attention, for the purpose of preventing the premature failure. In past decades, reinforcements on conventional metallic structures and composite structures have been proposed, such as the bonded patch and tear strap method, as well as the interlaminar doubler solution. The bonded tear strap method was also adopted in FML [13], and on basis of it an internal tear strap concept was proposed benefiting from the hybrid nature of FML [14]. Moreover, a structure termed as CentrAl has also been developed [15]. It should be noted that the present investigations were only focused on the improvement of fatigue resistance of FML, while almost no studies about the reinforcement of notched FML have been carried out.

Based on the previous investigations, the bonded patch reinforced concept was employed in notched GLARE in present paper. Mechanical response and damage behavior of both the single patch reinforced GLARE (SPR-GLARE) and double patches reinforced GLARE (DPR-GLARE) were investigated. For reference, those of the standard GLARE were also presented. Accordingly, the reinforced effect of titanium patch on notched behavior of GLARE laminate was clearly illustrated. 


\section{Experimental procedures}

\subsection{Materials and specimens}

The FML under investigation in present paper is the standard GLARE 3-3/2 laminate, in which three 2024-T3 aluminium layers and two S4C9-glass/SY-24-epoxy glass fiber reinforced epoxy composite (GFRP) layers were alternatively bonded up, and each GFRP layer consisted two cross-ply laminas. Thus a symmetric configuration was obtained for GLARE 3-3/2 laminate, which was given as $[\mathrm{Al} / 0 / 90 / \mathrm{Al} / 90 / 0 / \mathrm{Al}]$, as shown in Figure 1. In order to achieve a well bonding performance, the aluminium sheets were treated by means of the phosphoric acid anodizing method following the ASTM D3933, and after drying an epoxy primer was sprayed on them. The stacked laminates were cured in an autoclave at $120^{\circ} \mathrm{C}$ for two hours, where a heating rate of $2{ }^{\circ} \mathrm{C} / \mathrm{min}$ was adopted. At approximately $70{ }^{\circ} \mathrm{C}$, the autoclave was pressured to $0.5 \mathrm{MPa}$, and it was maintained till the end of the curing process. It should be noted that, the GLARE laminate under curing was kept in a vacuum bag with a vacuum degree of $0.1 \mathrm{MPa}$.

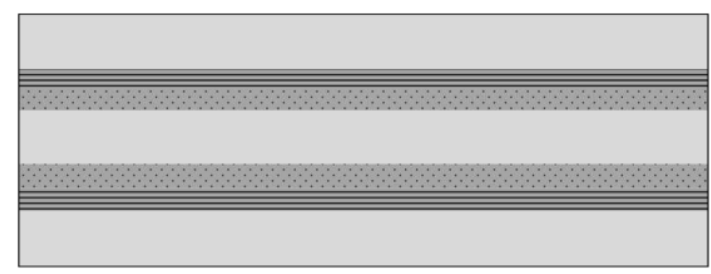

Aluminum $\equiv 0^{\circ}$ GFRP $90^{\circ}$ GFRP

Figure 1. Configuration of the GLARE 3-3/2 laminate.

All test specimens were cut from the GLARE panels with dimensions of $370 \mathrm{~mm} \times 370 \mathrm{~mm}$ by using the water jet cutting method. The length $(L)$ and width $(W)$ of the specimen was $250 \mathrm{~mm}$ and $25 \mathrm{~mm}$, respectively, as well as the thickness $(t)$ was $1.8 \mathrm{~mm}$, which was calculated based on the thickness of the constituents $(0.4 \mathrm{~mm}$ for aluminium sheet and $0.15 \mathrm{~mm}$ for GFRP lamina). Circular open hole with a diameter $(d)$ of $5 \mathrm{~mm}$ in the center of the specimen was also made by the water jet cutting method. Titanium patches with diameters $\left(d_{\mathrm{r}}\right)$ of $10 \mathrm{~mm}, 12.5 \mathrm{~mm}, 15 \mathrm{~mm}, 17.5 \mathrm{~mm}$ and $20 \mathrm{~mm}$ were cut from a $0.57 \mathrm{~mm}$ titanium sheet with using the wire electrical discharge machining method, and center circular holes with the same diameter as that in GLARE specimen were also made. The titanium patches were bonded on single or double sides of the GLARE specimens, thus two different reinforced structures were obtained, referred to as the single patch reinforced GLARE and double patch reinforced GLARE, respectively. The adhesive used for bonding the patch was an epoxy film adhesive (J272), which should be cured at $120^{\circ} \mathrm{C}$. Prior to bonding, the patches and GLARE specimens were well roughed with the sandpaper. The geometries and dimensions of the titanium reinforced GLARE specimens are shown in Figure 2, in which the $x$ direction denotes the longitudinal or $0^{\circ}$ fiber direction, while the $y$ direction denotes the lateral or $90^{\circ}$ fiber direction.
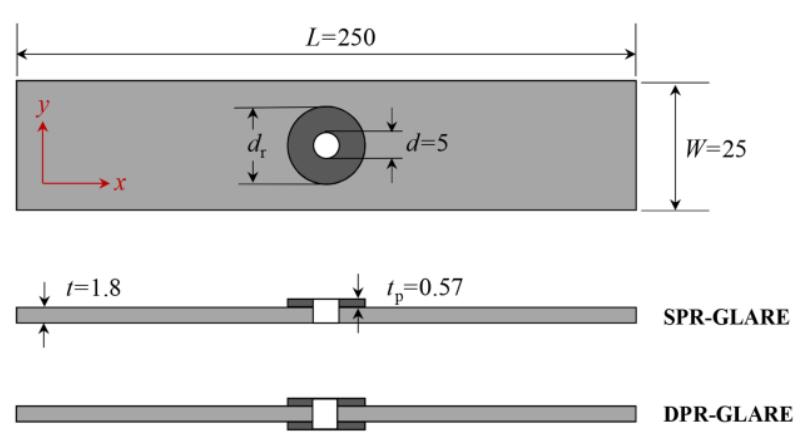

Figure 2. Geometries and dimensions of titanium reinforced GLARE specimens (dimensions in $\mathrm{mm}$ ).

\subsection{Test methods}

Quasi-static tensile tests were conducted on the titanium patch reinforced GLARE specimens at room temperature with employing a $50 \mathrm{kN}$ INSTRON 5569 testing machine. For reference, the standard or non-reinforced GLARE (NR-GLARE) specimen was also tested. Tensile load was applied with displacement control in $x$ direction at a cross-head speed of $1.0 \mathrm{~mm} / \mathrm{min}$, following the standard ASTM D3039. The displacement in the loading direction was measured with the LE-05 laser extensometer, in which the gauge length was specified to stride over the local reinforced region.

After test, the damage behaviour of the adhesive films between patches and GLARE laminates were examined optically with employing a Canon 700D camera. In addition, the outer aluminium layers of the post-failure specimens were chemically removed by means of the etching method, which was carried out in a sodium hydroxide solution. After this etching process, the damage patterns of the GFRP layers were examined, for the purpose of illustrating the effects of bonded patches and their dimensions on failure behaviour of the GLARE laminates.

\section{Results and discussion}

\subsection{Stress-strain relationships}

In order to evaluate the reinforced effect of titanium patch on mechanical response of GLARE laminate, the stressstrain curves of SRP-GLARE and DPR-GLARE were firstly investigated, as shown in Figure 3. For reference, that of the standard GLARE or NR-GLARE is also present. It should be noted that the stress mentioned here is the gross stress, which is calculated by dividing the applied load by the overall width of the specimen. Similarly, the notched strength discussed in later sections is the gross notched strength.

As can be seen that, the stress-strain curves of the reinforced GLARE laminates present a typical bilinear behaviour as that of the NR-GLARE showed, in spite of the slight descending in the second parts which are marked in the dashed boxes in Figure 3. Unlike the stress-strain curve of the unnotched GLARE laminate, slow transition zones between the first and second parts are detected in stress-strain curves of these notched 
specimens, which correspond to the local plastic deformations of aluminium in the net sections that resulted from the stress concentration in the vicinities of the holes.

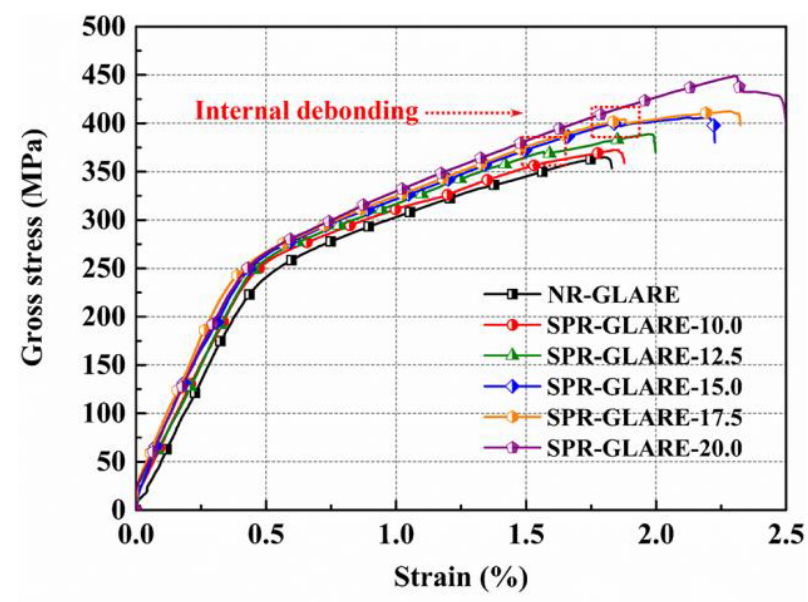

(a)

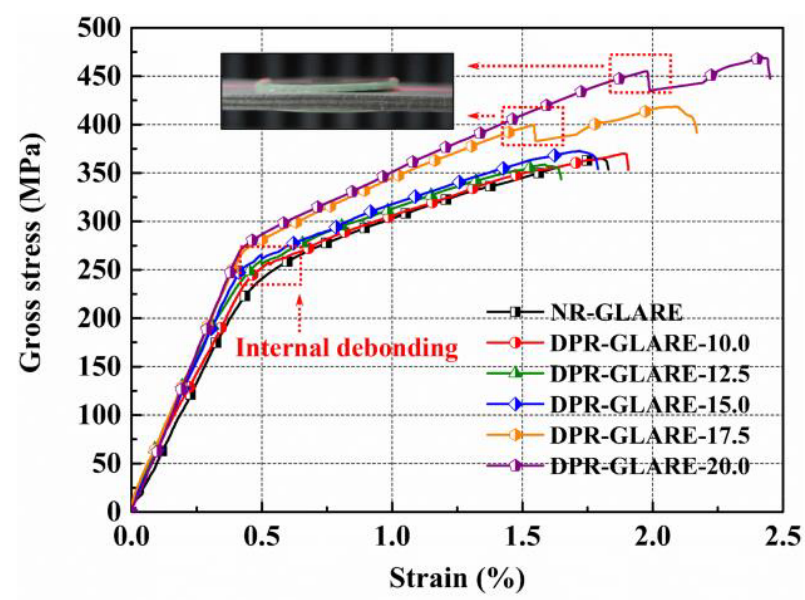

(b)

Figure 3. Typical stress-strain curves of (a) SPR-GLARE and (b) DPR-GLARE.

In addition, it can be seen that remarkable increase of the elastic modulus and yield strength are obtained by the reinforced GLARE laminates, especially for the DPRGLARE with large titanium patches. Moreover, the tangent modulus in second parts of the stress-strain curves increase as well, meanwhile significant increase of the ultimate strain is also detected. The increase of modulus and ultimate strain is attributed to that the load applied on the GLARE specimen is partly shared by the titanium patch, benefiting from its relative higher elastic modulus as compared with those of the constituents in GLARE laminates.

However, as observed from Figure 3(b), almost no increase of the tangent modulus (in second parts) and ultimate strain are achieved by DPR-GLARE with small patches $(10 \mathrm{~mm}, 12.5 \mathrm{~mm}$ and $15 \mathrm{~mm})$. It indicates that less work has been done by small patches in the later loading stage, namely that debonding between these small patches and GLARE laminates are preliminarily encountered. As expected, slight descending of the stressstrain curves are detected just after the elastic parts in
Figure 3(b), which are also audible in experiment. Note that this debonding is an internal case, for which cannot be detected visually in experiment. Similarly, the slight descending of stress-strain curves of SPR-GLARE with large patches are also caused by this internal debonding. For DPR-GLARE with larger patches, obvious descending of the stress-strain curves are detected just before fracture. They are associated with the edge debonding, as observed from the small image in Figure 3(b), which was captured during the experiment. It is interesting to note that significant rise of the stress-strain curves are detected after these descending, while those of the SPR-GLARE are negligible, which suggests that the double patch reinforced method is more effective.

\subsection{Notched strength}

For further evaluating the reinforced effect of titanium patch on mechanical response of notched GLARE laminates, the notched strength of SPR-GLARE and DPR-GLARE were compared and their corresponding strength increasing percentages over the NR-GLARE were also present, as shown in Figure 4.

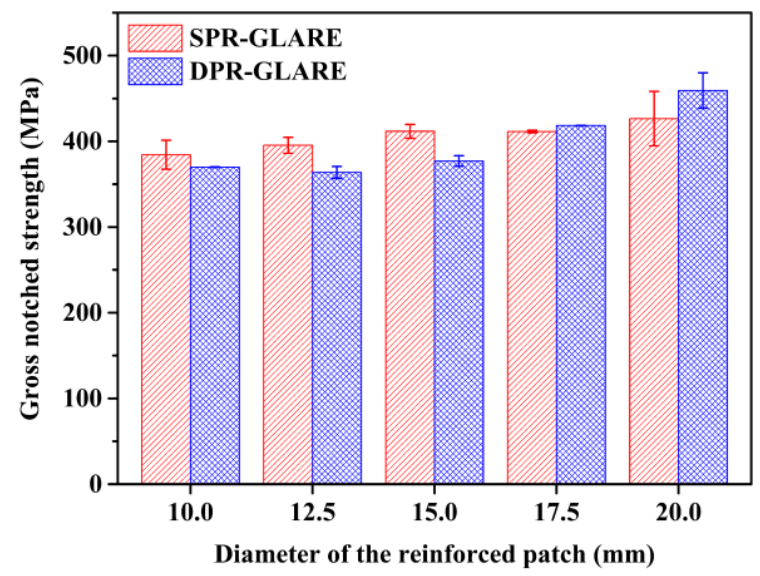

(a)

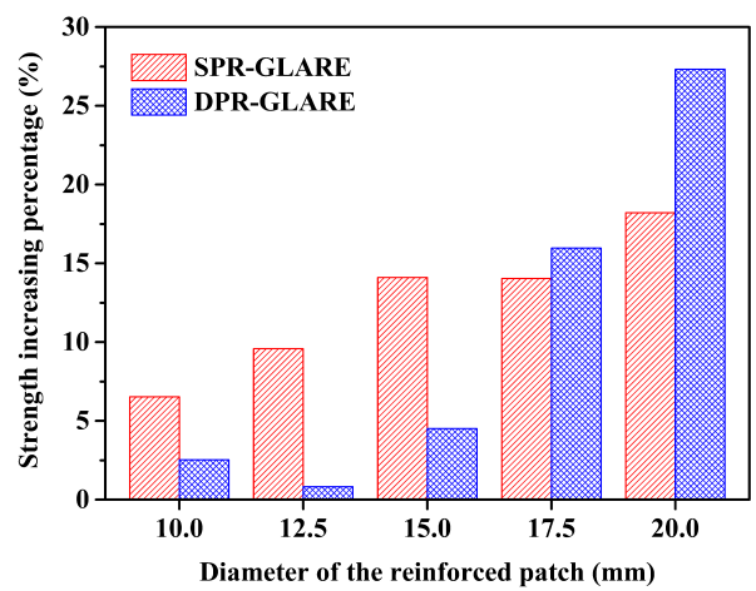

(b)

Figure 4. (a) Notched strength of reinforced GLARE and (b) the corresponding strength increasing percentages.

It can be seen from Figure 4, general upward trends of notched strength with increasing the patch diameter are obtained by both the SPR-GLARE and DPR-GLARE. 
When small patches (10 mm, $12.5 \mathrm{~mm}$ and $15 \mathrm{~mm})$ are adopted, the reinforced effects of titanium patches on notched strength of GLARE laminates are more significant for the single patch reinforced cases, while tiny strength increasing percentages are obtained by the DPR-GLARE, which are less than 5\%. This phenomenon is in contradiction with the general mind, which may be attributed to the introduction of thermal residual stress, for which the bonding process of titanium patch on GLARE laminate was operated at an elevated temperature of $120^{\circ} \mathrm{C}$, as well as their thermal expansion coefficients are mismatch. For double patch reinforced case, the thermal residual stress effect is more remarkable, thus the reinforced effect is seriously weakened.

As the patch diameter exceeds $17.5 \mathrm{~mm}$, the reinforced effect of DPR-GLARE becomes more prominent, and a strength increasing percentage of $27.31 \%$ is obtained by the DPR-GLARE with two $20 \mathrm{~mm}$ titanium patches. While less increase of the notched strength of SPR-GLARE is achieved as further increasing the patch diameter from $15 \mathrm{~mm}$ to $20 \mathrm{~mm}$. With the increasing of patch diameter, the thermal residual stress effect becomes negligible as compared with the significant increase of local stiffness in the reinforced region, accordingly a higher load bearing capacity will be achieved and which is more remarkable for DPR-GLARE. In addition, due to the asymmetric configuration of SPRGLARE, a bending effect will be introduced at the application of external load and it becomes more serious as the patch diameter increasing. The bending effect will constitute a risk to the loading bearing capacity of the SPR-GLARE, since it can result in an increase of the peeling stress in the adhesive film, as well as a higher tensile stress can be formed at the un-reinforced side of the laminate. Based on the above discussions, it should be noted that attention must be paid on both the thermal residual stress and bending effect when the secondary cocure reinforcement is adopted.

\subsection{Damage behavior}

For the purpose of illustrating the reinforced mechanism of titanium patch on GLARE laminate. The damage behavior of reinforced GLARE which played a dominant role in the mechanical response was emphatically investigated in present paper. Firstly, the failure behavior of the adhesive film between the patch and GLARE laminate was examined. It is of utmost importance, since the adhesive function as a medium for load transferring between the patch and GLARE laminate, accordingly it will be the most direct reflection of the reinforced effect of titanium patch. Secondly, the damage behavior of the GLARE laminate was characterized, it mainly focused on that of the inner GFRP layer, for which was the determinant of the final fracture of the reinforced GLARE.

\subsubsection{Damage behavior of adhesive}

Figure 5 shows the damage behavior of adhesive films between titanium patches and GLARE laminates in SPRGLARE and DPR-GLARE.
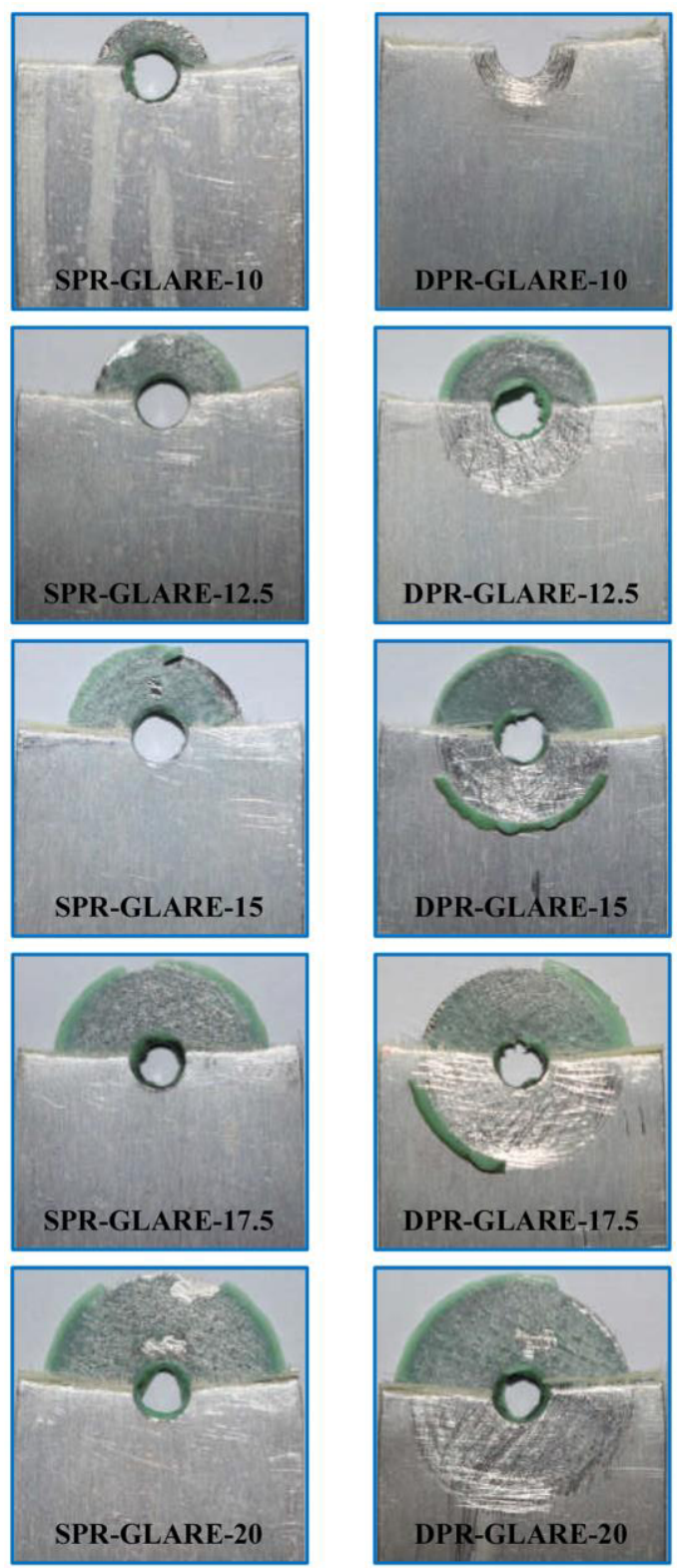

(a)

(b)

Figure 5. Damage behavior of the adhesive between patch and GLARE laminate: (a) SPR-GLARE; (b) DPR-GLARE.

As observed from Figure 5 (a), the damage patterns of adhesive films between patches and GLARE laminates in SPR-GLARE are characterized by the combination of adhesive failure and cohesive failure, which denote respectively the weak and strong load transfer capacity. For small patch case, the cohesive failure occurs at the free edge of the patch, it is also detected in the vicinity of the hole when the patch is large enough, as well as some punctate cohesive failure can also be detected. Positions where cohesive failure take place suggest that higher shear stress and peel stress present at the free edge of the patch, as well as in the vicinity of the hole, which is similar to that in previous investigation [16]. 
For DPR-GLARE with small patches, typical adhesive failure behavior are detected, while for large patch cases the same cohesive failure as that in SPRGLARE are obtained. The adhesive failure present in small patch case suggests that less applied load has been born by the titanium patch, and it is the reason that results in the weak reinforced effect, as above described in Figure 4. This phenomenon is also related with the thermal residual stress, as well as with the smaller bonding area. Consequently, it can be concluded that the bonding performance is another critical factor that associates with the reinforced effect.

\subsubsection{Damage behavior of the GLARE laminate}

Figure 6 shows the damage behavior of GLARE laminates in SPR-GLARE and DPR-GLARE, in which the damage patterns are examined after removing the outer aluminium sheets by means of the chemical etching method. As can be seen from Figure 6, damage patterns of the reinforced GLARE are similar to that of the standard one, which are usually characterized by the fiber breakage, splitting and delamination, and the delamination size is consistent with the splitting level. However, the degree of splitting or delamination is different between SPR-GLAER and DPR-GLARE, as well as between different patch diameter conditions.

For DPR-GLARE, a triangular delamination shape is detected, regardless of the patch diameter. The delamination sizes of DPR-GLARE with patches less than $15 \mathrm{~mm}$ are the same with that of the standard GLARE laminate, but significant increase of the delamination size can be obtained with increasing the patch diameter to $17.5 \mathrm{~mm}$ and $20 \mathrm{~mm}$. As previous investigation showed, the delamination was benefit for stress redistribution, namely that a larger delamination will suggest a higher load bearing capacity [17]. It is in accordance with the variation of the notch strength present in Figure 4, where negligible strength increasing ratio is obtained for DPR-GLARE with small patch while it is more remarkable when the patch is large enough. Similar upward trend is obtained by the delamination size of SPR-GLARE when increasing the patch diameter, however an analogous trapezoid delamination shape is detected, which is more serious than that in DPR-GLARE, especially for the large patch cases. It is mainly attributed to the bending effect that resulted from the asymmetric configuration, which has introduced a higher out-of-plane stress. It should be noted that the delamination caused by the bending effect plays a less role in stress redistribution.
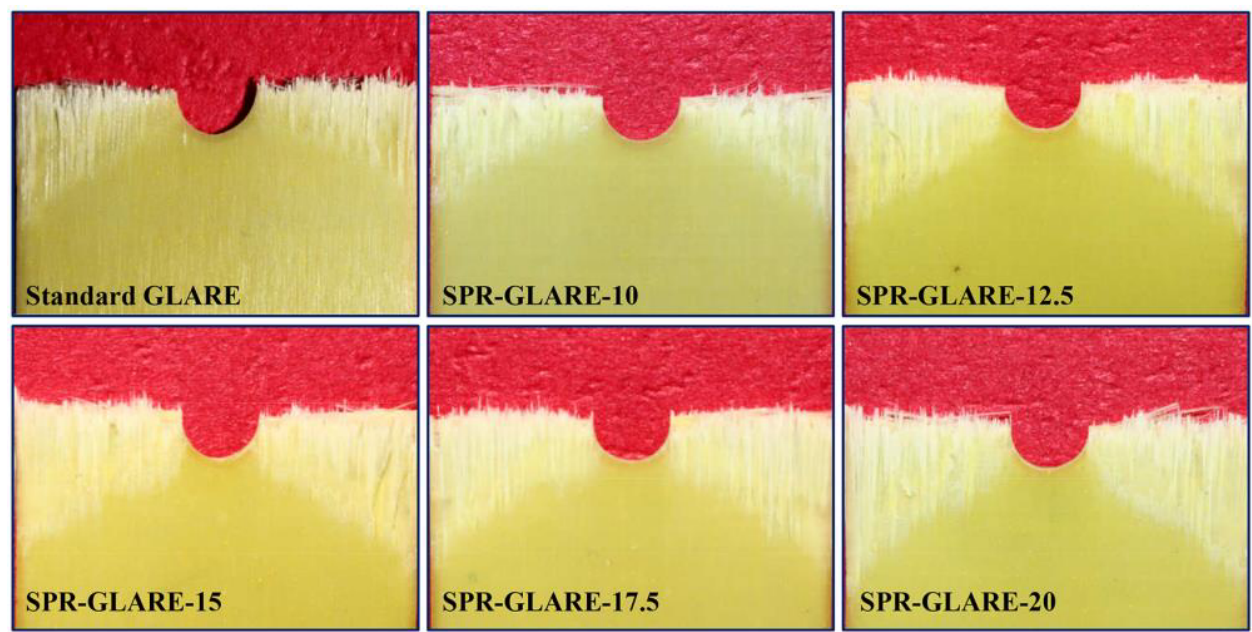

(a)
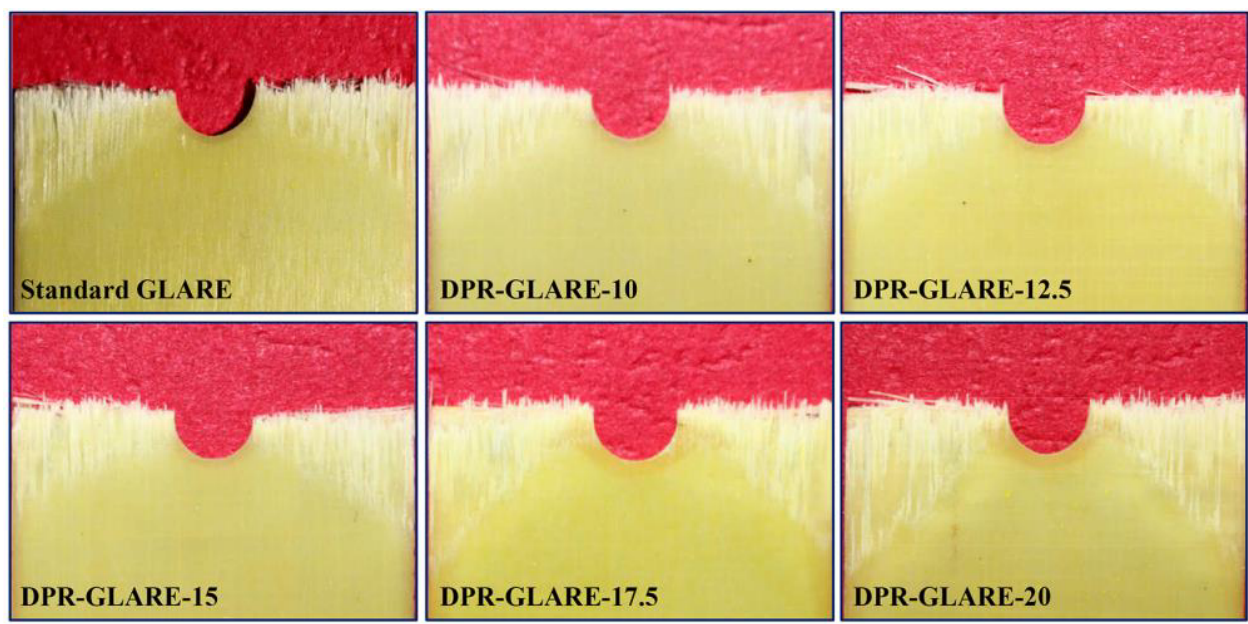

(b)

Figure 6. Damage behavior of the GLARE laminate: (a) SPR-GLARE; (b) DPR-GLARE. 


\section{Conclusions}

The purpose of present paper is to investigate the reinforced effect of titanium patch on notched behavior of GLARE laminate. The mechanical response analyses revealed that the modulus and yield strength of GLARE laminate were increased by bonding the titanium patches. As well as significant increase of notched strength was obtained, especially for the DPR-GLARE with $20 \mathrm{~mm}$ patches, which has achieved a strength increasing percentage of $27.31 \%$. However, negligible reinforced effect could be obtained by DPR-GLARE with small patches due to the thermal residual stress effect. Moreover the notched strength of SPR-GLARE increased less when the patch diameter reached to some certain values, which was attributed to the bending effect. The comparison suggests that the thermal residual stress and bending effects are of utmost importance in the case of secondary cocure reinforcement, and they will be further investigated experimentally or numerically in our future work.

In addition, the damage behavior of the adhesive between patch and GLARE laminate was examined, which was usually characterized by adhesive failure for SPR-GLARE and DPR-GLARE with large patches, while an adhesive failure was obtained by DPR-GLARE with small patch, which was associated with the negligible reinforced effect of titanium on mechanical response. Furthermore, the damage behavior of GLARE laminate was examined later, where the delamination sizes of SPR-GLARE and DPR-GLARE both increased with increasing the patch diameters, which was benefit for the strength improving. Meanwhile undesired trapezoid delamination was also detected in SPR-GLARE with large patche due to the bending effect.

It is expected that present investigations can provide some useful information on further structural design and application of the FMLs in aeronautics.

\section{Acknowledgement}

The present work was supported by the National Science Foundation of China under Grant Nos. 51271067, 11272105 and 11572101 .

\section{References}

1. R.C. Alderliesten, Mater. Des. 66, 421 (2015)

2. T. Sinmazçelik, E. Avcu, M.Ö. Bora, O. Çoban, Mater. Des. 32, 3671 (2011)

3. H. Nakatani, T. Kosaka, K. Osaka, Y. Sawada, Compos. Part A 42, 772 (2011)

4. P. Manikandan, G.B. Chai, Compos. Struct. 117, 17 (2014)

5. Y.C. Zhong, C.S. Joshi, Mater. Des. 87, 138 (2015)

6. E. Sadeghpour, M. Sadighi, S. Dariushi, J. Reinf. Plast. Compos. 32, 1143 (2013)

7. G.C. Wu, Y. Tan, J.M. Yang, Mater. Sci. Eng. A 457, 338 (2007)

8. M. Hagenbeek, C. Van Hengel, O.J. Bosker, C.A.J.R. Vermeeren, Appl. Compos. Mater. 10, 207 (2003)

9. M. Kawai, Y. Arai, Compos. Part A 12, 1990 (2009)

10. I. Lapczyk, J.A. Hurtado, Compos. Part A 11, 2333 (2007)

11. J.F. Chen, E. Morozov, K. Shankar, Compos. Struct. 114, 64 (2014)

12. D.D. Du, Y.B. Hu, H.G. Li, C. Liu, J. Tao, Compos. Part B 91, 65 (2016)

13. R. Rodi, R.C. Alderliesten, R. Benedictus, The 24th ICAF Symposium (Naples, Italy, 2007)

14. C. Rans, F. Moriniere, R. Rodi, R.C. Alderliesten, R. Benedictus, J. Aircraft 48, 2122 (2011)

15. G.H.J.J. Roebroeks, P.A. Hooijmeijer, E.J. Kroon, M.B. Heinimann, First International Conference on Damage Tolerance of Aircraft Structures (Delft, Netherland, 2007)

16. R.D.S.G. Campilho, M.F.S.F. de Moura, J.J.M.S. Domingues, Compos. Sci. Technol. 65, 1948 (2005)

17. G.D. Lawcock, L. Ye, Y.W. Mai, C.T. Sun, Compos. Sci. Technol. 57, 1609 (1998) 